Protocol paper

\title{
Resistance training programs on bone related variables and functional independence of postmenopausal women in pharmacological treatment: A randomized controlled trial
} \author{
Rodrigo Gomes de Souza Vale ${ }^{\mathrm{d}}$, Alexandre Janotta Drigo ${ }^{\mathrm{e}}$, \\ Nébia Maria Almeida de Figueiredo ${ }^{\mathrm{a}}$

 \\ ${ }^{\mathrm{b}}$ Federal Institute of Education, Science and Technology (IFPA), Campus de Tucuruí, Brazil \\ ${ }^{\mathrm{c}}$ Pará State University (UEPA), Campus de Tucuruí, Brazil \\ ' State University of Rio de Janeiro (UERJ), Rio de Janeiro, Brazil \\ e São Paulo State University "Julio de Mesquita Filho" (UNESP-RC), Campus Rio Claro, Brazil \\ ${ }^{\mathrm{f}}$ Pedro II School, Rio de Janeiro, Brazil \\ ${ }^{\mathrm{g}}$ Autonomous University of Baja California, Mexico
}

Claudio Joaquim Borba-Pinheiro ${ }^{a, b, c, *}$, Estélio Henrique Martin Dantas ${ }^{\mathrm{a}}$, Mauro César Gurgel de Alencar Carvalho ${ }^{\mathrm{f}}$, Teresa Tonini ${ }^{\mathrm{a}}$, Edgar Ismael Alarcon Meza ${ }^{\mathrm{g}}$,

\section{A R T I C L E I N F O}

\section{Article history:}

Received 6 August 2015

Received in revised form 12 February 2016

Accepted 13 February 2016

Available online 3 March 2016

\section{Keywords:}

Bone density

Physical function

Resistance exercise

Quality of life

Muscle strength

\begin{abstract}
A B S T R A C T
Introduction: Osteoporosis is a chronic disease that leads to bone fragility and is associated with fracture risks and serious consequences for mobility.

Objective: To verify the effects of two linear programs of resistance training (RT) on bone mineral density (BMD), functional autonomy (FA), muscular strength and quality of life (QoL) of postmenopausal women in pharmacological treatment.

Study design: Randomized controlled trial, code: RBR-6bqsw8.

Methods: 52 volunteers were distributed into three groups, according to randomly parallel form: RT3times-per-week (RT3, $n=20$ ); RT2times-per-week (RT2, $n=16$ ) and control group (CG, $n=16)$. The following assessment tools were used: bone mineral density (BMD) by dual X-ray absorptiometry, 'Latin America Group for maturity' (GDLAM) protocol for FA, 10RM test for leg exercises and the 'Osteoporosis Assessment Questionnaire' (OPAQ) for QoL. The physical activities were planned for 13 months in cycles with different intensities. A two-way ANOVA with Bonferroni post-hoc test were used.

Results: The results showed that the RT3/week was significantly more efficient $(p<0.05)$ compared with RT2/week, including: All BMD variables, FA $(\Delta \%=29.3 \%)$, leg press at $45^{\circ}(\Delta \%=24.97 \%)$ and OPAQ ( $\Delta \%=20.23 \%$ ). In addition, both RT3 and RT2 groups were more efficient $(p<0.05)$ compared with CG, including: total BMD $(\Delta \%=0.09 \%)$ and $(\Delta \%=0.06 \%) ; \operatorname{FA}(\Delta \%=7.1 \%)$ and RT2 $(\Delta \%=3.78 \%)$; Leg press at $45^{\circ}$ $(\Delta \%=84.1 \%)$ and $(\Delta \%=59.1 \%)$; keen extension $(\Delta \%=15.28 \%)$ and $(\Delta \%=20.37 \%)$; OPAQ $(\Delta \%=57.61 \%)$ and $(\Delta \%=37.37 \%)$, respectively.

Conclusion: The study showed that both experimental groups presented favorable results for BMD, strength, FA and QoL. However, the RT3 showed the best results compared to other groups after 13 months of intervention.
\end{abstract}

(c) 2016 Elsevier Ireland Ltd. All rights reserved.

\footnotetext{
* Corresponding author at: Canada Street Number 40, Vila Permanente District, Tucuruí, PA 68464 000, Brazil.

E-mail addresses: claudioborba18@gmail.com, borba.pinheiro@ifpa.edu.br (C.J. Borba-Pinheiro).
}

\section{Introduction}

The need for maintenance of physical activity (PA) throughout life is widely recommended by the scientific literature, in particular, during the stage at which aging accentuates the decline of the systems responsible for the functionality of the body, thus increasing the risk of developing diseases with physical and 
psychological consequences (Cabral et al., 2014; Dantas, Figueira, Emygdio, \& Vale, 2014).

Osteoporosis is a chronic disease of the skeletal system that leads to bone fragility and is associated with fracture risks and serious consequences for mobility (Kanis et al., 2013). In the elderly, bone fragility, sarcopenia, the risk of falls and the frequency of falls are the decisive factors that considerably affect the quality of life (QoL) (Kanis et al., 2013; Patel, Tweed, \& Chinappen, 2005); moreover, these factors also affect the ability of the elderly to participate in daily activities, which consequently affects their functional autonomy (FA) (Cabral et al., 2014; Dantas et al., 2014; Kanis et al., 2013).

The women are more likely owing to the endogenous release of sex hormones and the absorption of the mineral calcium (Ca) (Kanis et al., 2013; Pinheiro, Ciconelli, Jacques et al., 2010). In addition to gender, other risk factors associated with osteoporosis include genetic inheritance, European descent, age, calcium deficiency, excessive consumption of tobacco and alcohol, diseases and medications related to low bone mineral density (BMD) and physical inactivity (Pinheiro, Ciconelli, Jacques et al., 2010). Among women, other risk factors were associated to recurrent falls: age, previous fracture, sedentary lifestyle, poor QoL, diabetes mellitus and current use of benzodiazepine (Kanis et al., 2013; Pinheiro, Ciconelli, Martini et al., 2010).

Pharmacological treatment, with the use of alendronate (bisphosphonate), also efficiently controls the bone loss, acting as an inhibitor of the bone resorption caused by the osteoclastic action (Kanis et al., 2013; Langdahl, 2011).

Considering all of the risk factors, special attention should be given to physical inactivity. The appropriate regular PA is recommended for their beneficial effects on BMD and for the maintenance FA and QoL (Park et al., 2013; Pinheiro, Ciconelli, Jacques et al., 2010).
The relationship between low BMD and PA level was first suggested in the 1940s by the related case of osteoporosis in an immobilized boy (Albright, Burnett, Cope, \& Parson, 1941). In contrary, bone mass in athletes who weight train is greater than athletes who do not weight train. This can be explained because the effect of exercise on bone is thought to be due to a net increase in bone formation (Park et al., 2013; Rubin, Judex, \& Qin, 2006).

In this study, special attention was given to PA because it has been considered a potential factor to both treatments: pharmacological associated and not associated with PA in this specific problem (Rubin et al., 2006). Given the different types of PAs, resistance training (RT) has been cited as an effective for the treatment of osteoporosis and sarcopenia; due to the increase in muscular strength, FA, QoL and BMD (Cabral et al., 2014; Cusller et al., 2005).

Considering the above, this study aimed to verify the effects of two linear programs of RT on BMD, FA, muscular strength and QoL of postmenopausal women in pharmacological treatment. Our major hypothesis is based on the assumption that resistance training 3 times per week results in more pronounced stimuli than resistance training 2 times per week and should be more effective in maintaining or increasing bone density in postmenopausal women with pharmacological treatment compared to controls with pharmacological treatment without exercise.

\section{Material and methods}

\subsection{Participants}

The volunteers residing in Tucuruí (Pará-Brazil) were recruited via local radio and television programs. Registrations of volunteers were performed in campus XIII of State Pará University in the municipality of Tucuruí. The participants performed diagnostic

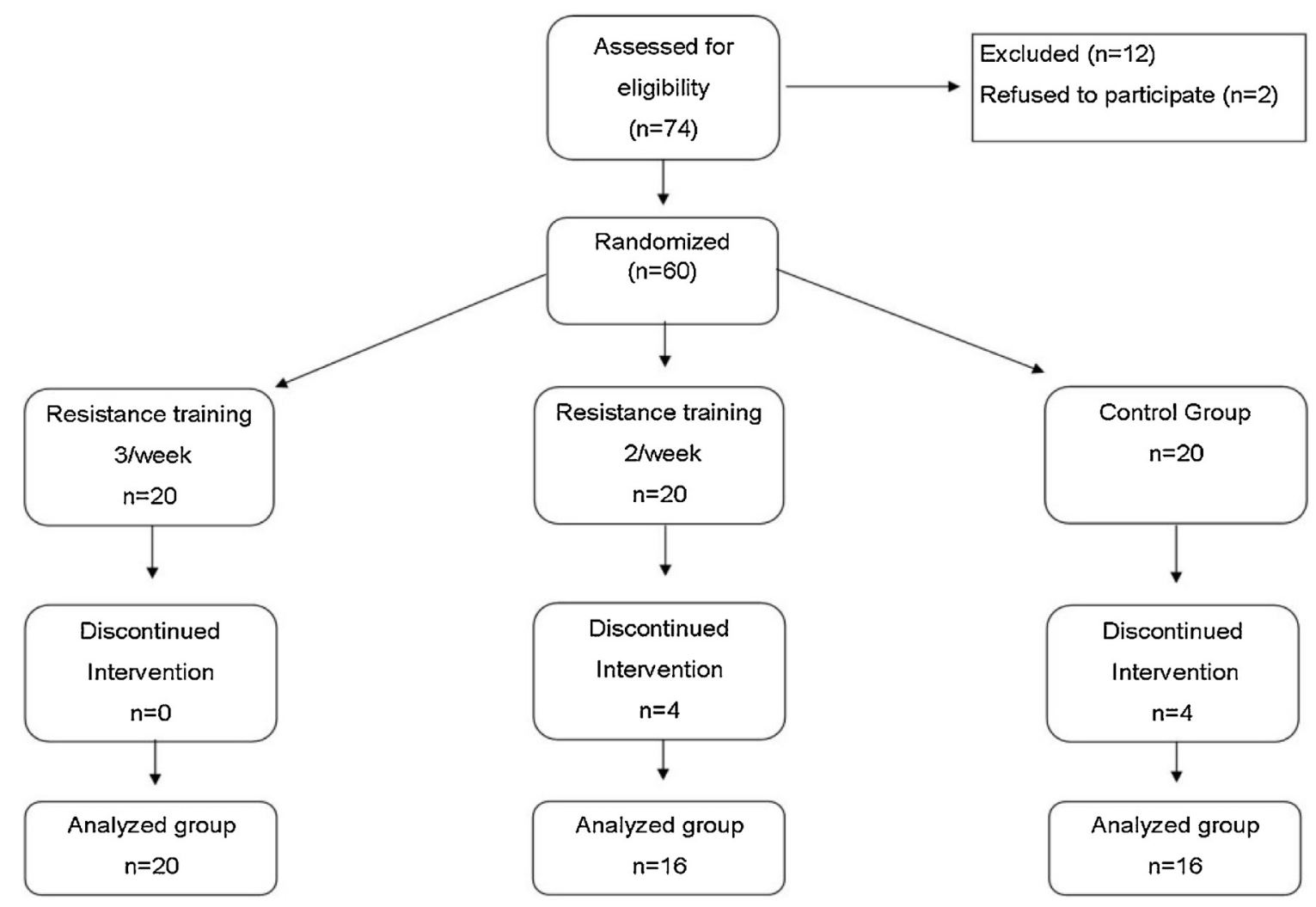

Fig. 1. Diagram of the sample selection process for this study. 
assessments through interview and blood pressure check in time period 8:00 to 10:00 from Monday to Friday of the January month in 2012. The following Inclusion and exclusion criteria for this study are listed.

Inclusion criteria: (a) female volunteers; (b) aged over 50 years; (c) with low BMD: T - score < -1SD (low bone density); (d) different ethnic population (descendants of Europeans, Blacks and Indians); (e) patients being treated with sodium alendronate [70 mg] and/or vitamin $\mathrm{D}_{3}$ [5600 IU]; (f) postmenopausal individuals (self-declared); (g) no previous history of fractures (selfdeclared); (h) no history for at least 1 year of regular practice of physical activity; (i) with indication/medical clearance for physical exercises practices.

Exclusion criteria: (a) who made some sort of surgery in the last six months; (b) who had early menopause by ovary removal; (c) with uncontrolled hypertension; (d) that use additional bone active medications or hormone replacement therapy; and (e) Individuals who were being treated with drugs that induces low BMD, as glucocorticoids.

It was used a mathematical calculation to verifier the sample size (Barreto \& Ribeiro, 2004) after previous observations (BorbaPinheiro et al., 2010). We expected a change of $0.5-4 \%$ in the bone formation by DXA assessment, as an indicator of osteogenic response from training and $5 \%$ or more for others variables of the study. With a power of $80 \%$ and significance level of $5 \%$, 20 participants in each group were required to detect such a significant difference, considering a possible sample loss of $20 \%$ during the study. Thus, the minimum number of participants established for each group was of 16 subjects.

The selection process of this study is presented in Fig. 1.

The study sample comprised a total of 52 analyzed women volunteers with low BMD [25Caucasian, 20 of Afro-Brazilian and 7 of Indian-Brazilian descent]. Participants were distributed into two experimental groups and control group (CG) according to randomly parallel form: Resistance Training 3 times-per-week (RT3, $n=20)$; Resistance Training 2 times-per-week (RT2, $n=16$ ) and the control group (CG, $n=16$ ). The CG was drawn from the same sample of volunteer women and was made up of women who were encouraged not to practice regular PA during the study period (Fig. 1). The drop-outs was characterized by the not frequency in the training period (RT2, $n=4$ ) and evaluation tests (CG, $n=4)$ of dependent variables (Fig. 1). The study was approved by the Ethics Committee of the Federal State Rio de Janeiro University, Brazil, protocol number 0050/2011 FR 478507CAAE:0061.0.313.412-11, according to the standards mandated by the Declaration of Helsinki (World Medical Association, 2008).

\subsection{Assessment protocols}

\subsubsection{Assessment of bone mass by dual X-ray absorptiometry}

BMD was determined by scans Lunar ${ }^{\circledR}$ model DPX-L (USA). Variables obtained by DXA were: measures of BMD $\left[\mathrm{g} / \mathrm{cm}^{2}\right]$ values in the lumbar spine $\mathrm{L}_{2}-\mathrm{L}_{4}$ region and the right total femur. Collected data analyses were performed using the following software version: DPXL 4.7e. DXPL uses an X-ray source at $78 \mathrm{kVp}$ and a K-edge filter to produce stable beams of X-rays at energies of 38 and $70 \mathrm{keV}$. The radiation dose for a total body scan set on standard thickness using DPXL is $0.002 \mathrm{mSv}$. 4\% above of the radiation dose received in a chest X-ray. All scans were performed by a particular laboratory that did not know the purpose of the study in order to not have any influence on study. To determine osteoporosis levels, was observed [T scores] as a condition in which BMD measures lower than -2.5 times the standard deviation [SD] and osteopenia with values between -1 and -2.5 SD compared with mean of young people (Kanis et al., 2013).

\subsubsection{Assessment of functional autonomy}

The protocol of the Latin American Development Group for Maturity (GDLAM) aims to standardize the assessment of FA (Dantas et al., 2014). GDLAM protocol has the following tests to assess FA:

(a) $10-\mathrm{m}$ walk (10MW): walk the distance of $10 \mathrm{~m}$ rapidly to evaluate the displacement speed; (b) rising from sitting position (RSP): the individuals should get up and sit down of an chair five times consecutively to evaluate the strength of lower limbs; (c) rising from ventral decubitus position (RVDP): the individuals must stand up as fast as possible after the command to assess the ability to the rise quickly from the ground; (d) rising from a chair and walking around the house (RCWH): the test assess agility and dynamic balance; (e) putting on and taking off a shirt (PRTS): the individuals must put on and take off the shirt (G t-shirt Hering ${ }^{\circledR}$, Brazil) as fast as possible to assess flexibility. The GDLAM autonomy index (AI) was calculated using the following formula:

$\mathrm{AI}=\frac{[(10 \mathrm{mw}+\mathrm{RSP}+\mathrm{RVDP}+\mathrm{PRTS}) \times 2]+\mathrm{RCWH}}{4}$

For the performance of the tests, the following equipment was used: a tape measure (Sanny ${ }^{\mathbb{}}$, Brazil); a stopwatch (Casio ${ }^{\mathbb{R}}$, Brazil); a chair $50 \mathrm{~cm}$ above the ground; and a 20-mm-thick exercise mat.

\subsubsection{Assessment of muscular strength}

To assess muscle strength, the 10 maximum repetitions test (10MR) was used, in which the procedures adopted for the implementation were the following:

a Performance of static stretching to prepare the large muscle groups. Two to three series of exercises were performed with light loads for familiarization with the execution of the movements;

b Each individual had from three to four attempts to perform the test with a 10MR load according to the recommendations of the American College of Sports Medicine (American College Sports Medicine (ACSM), 2014). The breaks and the intervals - three to five minutes - between attempts were restoring. It is worth mentioning that the participants were not aware of the number of repetitions of the test, i.e., 10MR. The equipment used was from Physicus ${ }^{\circledR}$ (Brazil) and the following exercises were done: leg press $45^{\circ}$ and knee extension for all groups.

\subsubsection{Assessment of quality of life}

The "Osteoporosis Assessment Questionnaire" (OPAQ) is a tool used to measure the QoL of people with low BMD. This study used the Brazilian-Portuguese OPAQ (Cantarelli, Szejnfeld, Oliveira, Ciconelli, \& Ferraz, 1999) that checks issues related to general health, mobility, walking and leaning, back pain, flexibility, personal care, housework, movement, fear of falling, social activities, support from family and friends, pain associated with osteoporosis, sleep, fatigue, work, tension level, humor, body image, independence and total OPAQ count from zero to 100 points. Where the zero point = lower QoL and 100 points = highest QoL.

\subsubsection{Pharmacological treatment}

The medication bisphosphonate sodium alendronate [70 mg] once weekly and Vitamin $\mathrm{D}_{3}$ [5600IU] once daily was used by volunteers that showed $T-$ Score $<-2.5$ SD [osteoporosis]. In addition the volunteers that showed $T$ - score among -1 and -2.5 SD [osteopenia] used only vitamin $\mathrm{D}_{3}$ [5600IU/day] to aid in treatment for reduced bone mass (Kanis et al., 2013; Langdahl, 2011) in agreement with their prescriptions. Thus, in the RT3 11 volunteers used only vitamin $\mathrm{D}_{3}$, and 9 volunteers used 
alendronate+vitamin $\mathrm{D}_{3}$. In the RT2 11 volunteers used only vitamin $D_{3}$, and 5 volunteers used alendronate + vitamin $D_{3}$. And, in the CG 10 volunteers used only vitamin $D_{3}$, and 6 volunteers used alendronate + vitamin $D_{3}$. In addition, the medicines were used by two years, before the study, for the volunteers with osteopenia and at least four years, before the study, for the volunteers with osteoporosis. The medicine was consumed in the form of tablets by the volunteers from both groups during the study period. The volunteers had the treatment according with their needs, prescribed by a medical doctor.

\subsection{Intervention}

The physical activity investigated was performed for 13 months in the linear periodization according to the recommendations of Borba-Pinheiro et al. (2010). The classes for experimental groups (RT3 and RT2), were conducted three and two times a week, respectively, on alternate days with each class lasting $60 \mathrm{~min}$. Equal periodization was used for both groups. The only difference was that one group performed the three times/week exercises and the other twice a week. At the beginning and end of the training sessions, were used 10s of stretches for each muscle group, used in the exercises machines.

For the RT groups, the 10 maximum repetitions [10MR] test was used to stipulate the maximum load for each cycle, and the intensities varied between $60 \%$ and $90 \%$. The periodization was carried with 7 monthly cycles [60\%,65\%, 70\%, 75\%, 80\%, 85\% and $90 \%$ ]; in addition 3 bimonthly cycles [70\%, 80\% and 90\%] of the values found in the 10MR test, totaling 13 months. All procedures of linear periodization including three sets per exercise, repetitions numbers/sets, rest intervals between exercises and sessions respected the scientific principle of inter-dependence volume $\mathrm{x}$ intensity (American College Sports Medicine (ACSM), 2014; Kraemer \& Fleck, 2009). The equipment used for the RT was from Pró-Physical (Brazil), and the following exercises were performed: leg press at $45^{\circ}$; knee extension; plantar flexion; squats; hip adduction; gluts (machine for gluts); elbow flexion; elbow extension; and shoulder adduction.

\subsection{Statistical analysis}

The software PASW ${ }^{\circledR} 20.0$ was used and the significance level of $p<0.05$ was adopted for the statistical analysis. Descriptive analysis was carried out with measures of central tendencies and dispersion. A Shapiro-Wilk and Levene's tests were performed to confirm proximity with a normal distribution and homoscedastic variance. One-Way ANOVA and Kruskal Wallis tests in the baseline analysis were used. The ANOVA with repeated measures was carried out in factors groups (RT3, RT2 and CG) and time (preand post-test) for analysis intra and inter-groups, and the Bonferroni post-hoc test was performed for multiple differences of variables between groups. In addition, the effect size was present for all variables. Specially, a Wilcoxon and Kruskal Wallis tests were carried for QoL analysis with Dunn post hoc test for multiple differences between groups. The equation $\Delta \%$ [(Posttest_test) $* 100 /$ test] was used to determine the percentage difference.

\section{Results}

Table 1 presents data on the descriptive and dependent baseline variables of the studied groups. In baseline, there were no statistical differences in age, age at menopause and for $T$ score related variables, BMD, FA and QoL. However, weight, BMI and frequency of weekly session showed differences $(p<0.05)$. In the weekly session this difference was expected because was a component of the training programs for between comparison of groups RT3 and RT2 (Table 1).

The power of the experiment presented to $\mathrm{L}_{2}-\mathrm{L}_{4}=94 \%$, neck femur $=98 \%$; besides, trochanter, total femur and total BMD $=99 \%$ showed strengthening the magnitude of results achieved in this analysis. Fig. 2 shows the results for BMD. The results show that RT3 presented intra-group improvements $(p<0.05)$ for all bone variables, as follows: $\mathrm{L}_{2}-\mathrm{L}_{4}(\Delta \%=0.07 \%)$; neck femur $(\Delta \%=0.12 \%)$, trochanter $(\Delta \%=0.09 \%)$, total femur $(\Delta \%=0.12 \%)$, total BMD $(\Delta \%=0.10 \%)$. And again, the RT3 showed better results $(p<0.05)$ compared to CG for BMD variables: Neck femur $(\Delta \%=0.12 \%)$, trochanter $(\Delta \%=0.08 \%)$; total femur $(\Delta \%=0.10 \%)$, total BMD ( $\Delta \%=0.09 \%$ ). In addition, the RT2 was statistically better compared

Table 1

The descriptive characteristics for volunteers groups in base line.

\begin{tabular}{|c|c|c|c|c|c|c|c|}
\hline \multicolumn{8}{|l|}{ Groups } \\
\hline \multirow[t]{2}{*}{ Variables } & \multicolumn{2}{|c|}{ RT3; $n=20$} & \multicolumn{2}{|c|}{$\mathrm{RT} 2 ; n=16$} & \multicolumn{2}{|c|}{ CG; $n=16$} & \multirow[t]{2}{*}{$\overline{p \text {-value }}$} \\
\hline & Mean & SD & Mean & SD & Mean & SD & \\
\hline Age (years) & 56.3 & 5.2 & 60.6 & 7.5 & 55.3 & 6.8 & 0.056 \\
\hline Menopause of age (years) & 44.4 & 4.1 & 44.8 & 2.8 & 45.7 & 3.4 & 0.661 \\
\hline Weight (kg) & 57.4 & 6.1 & 64.4 & 7.3 & 62.7 & 7.5 & 0.011 \\
\hline Height $(\mathrm{cm})$ & 149.05 & 0.03 & 149.06 & 0.06 & 150.06 & 0.05 & 0.808 \\
\hline $\operatorname{BMI}\left(\mathrm{kg} / \mathrm{m}^{2}\right)$ & 25.8 & 3.05 & 29.02 & 2.9 & 27.8 & 3.5 & 0.015 \\
\hline Frequency Session (class/week) & 2.58 & 0.11 & 1.62 & 0.09 & $*$ & $*$ & 0.001 \\
\hline T-Score $\mathrm{L}_{2}-\mathrm{L}_{4}(\mathrm{SD})$ & $-2,48$ & 0.89 & $-2,07$ & 0.66 & $-2,36$ & 0.37 & 0.221 \\
\hline T-Score Neck Femur (SD) & $-1,72$ & 0.71 & $-1,54$ & 0.91 & $-1,68$ & 0.97 & 0.821 \\
\hline T-Score Trochanter (DS) & $-1,09$ & 0.64 & $-0,99$ & 0.88 & $-1,02$ & 0.77 & 0.919 \\
\hline T-Score Total Femur (SD) & $-1,41$ & 0.64 & $-1,27$ & 0.89 & $-1,35$ & 0.85 & 0.872 \\
\hline BMD $\mathrm{L}_{2}-\mathrm{L}_{4}\left(\mathrm{~g} / \mathrm{cm}^{2}\right)$ & 0.908 & 0.11 & 0.958 & 0.07 & 0.913 & 0.06 & 0.167 \\
\hline BMD Neck Femur $\left(\mathrm{g} / \mathrm{cm}^{2}\right)$ & 0.768 & 0.08 & 0.790 & 0.12 & 0.786 & 0.11 & 0.788 \\
\hline BMD Trochanter $\left(\mathrm{g} / \mathrm{cm}^{2}\right)$ & 0.678 & 0.08 & 0.697 & 0.07 & 0.696 & 0.08 & 0.550 \\
\hline BMD Total Femur $\left(\mathrm{g} / \mathrm{cm}^{2}\right)$ & 0.714 & 0.07 & 0.738 & 0.08 & 0.732 & 0.09 & 0.707 \\
\hline BMD Total $\left(\mathrm{g} / \mathrm{cm}^{2}\right)$ & 0.777 & 0.06 & 0.796 & 0.06 & 0.793 & 0.07 & 0.410 \\
\hline Functional Autonomy Index (Score) & 28.42 & 3.53 & 28.13 & 2.99 & 28.17 & 2.18 & 0.953 \\
\hline Keen Extension Exercise (kg) & 28.55 & 6.46 & 30.38 & 04.06 & 28.75 & 4.77 & 0.553 \\
\hline Leg Press at $45^{\circ}$ Exercise (kg) & 82.25 & 12.96 & 73.81 & 13.46 & 78.81 & 16.23 & 0.217 \\
\hline OPAQ Total (points) & 330.20 & 22.02 & 313.31 & 22.01 & 312.31 & 35.94 & 0.090 \\
\hline
\end{tabular}

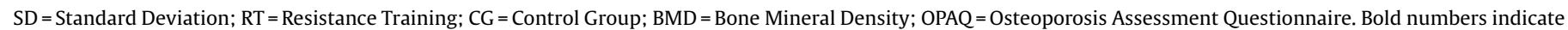
$p<0.05 .\left(^{*}\right)=$ No exercise frequency for CG. 


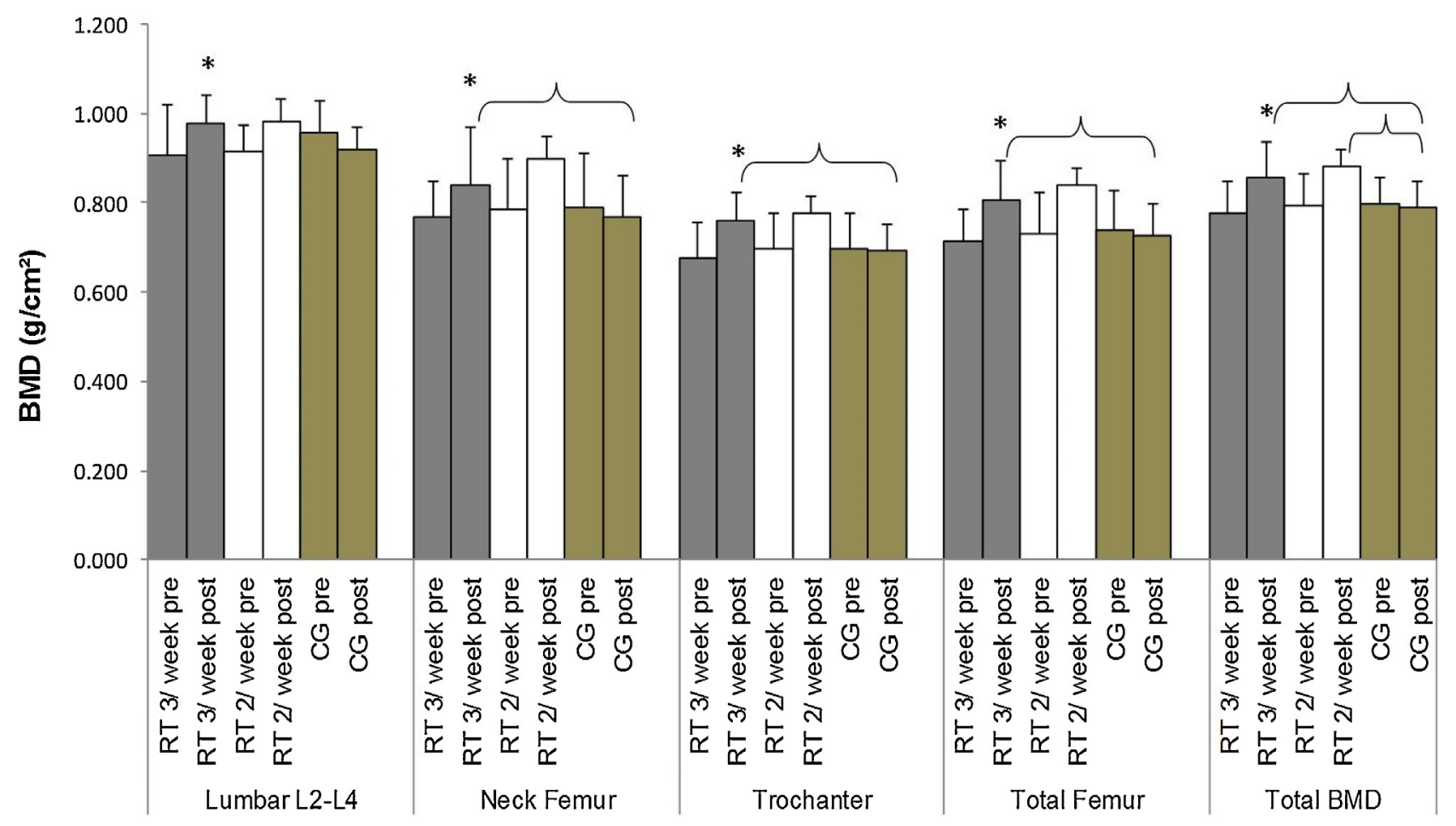

\section{Groups / Dependent BMD Variables and Tests}

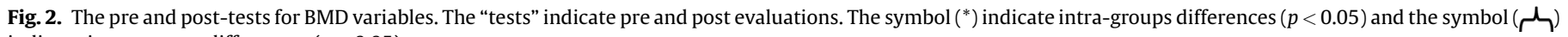
indicate inter-groups differences $(p<0.05)$.

with CG in total BMD $(\Delta \%=0.06 \%, \mathrm{p}=0.046)$. And, the CG remained with stable BMD (Fig. 2).

The power of the experiment presented to $A I=99 \%$ showed strengthening the magnitude of the analysis. Fig. 3 present the results for AI GDLAM protocol, and shows that both experimental groups were effective (intra-group $p<0.01$ ): RT3 ( $\Delta \%=7.58 \%$ ) and RT2 $(\Delta \%=4 \%)$. In addition, the RT3 showed better results than RT2
( $\Delta \%=29.3 \%)$. And also, both groups: RT3 $(\Delta \%=7.1 \%)$ and RT2 ( $\Delta \%=3.78 \%$ ) showed better results compared to the CG (Fig. 3 ).

The power of the experiment presented to leg press $45^{\circ}$ and knee extension $=99 \%$ showed strengthening the magnitude of this analysis. Fig. 4 shows the results for strength and also showed that both experimental groups were effective $(p<0.05$, intra-groups) for Leg Press $45^{\circ}$ : RT3 $(\Delta \%=78.85 \%)$ and RT2 $(\Delta \%=62.31 \%)$. The

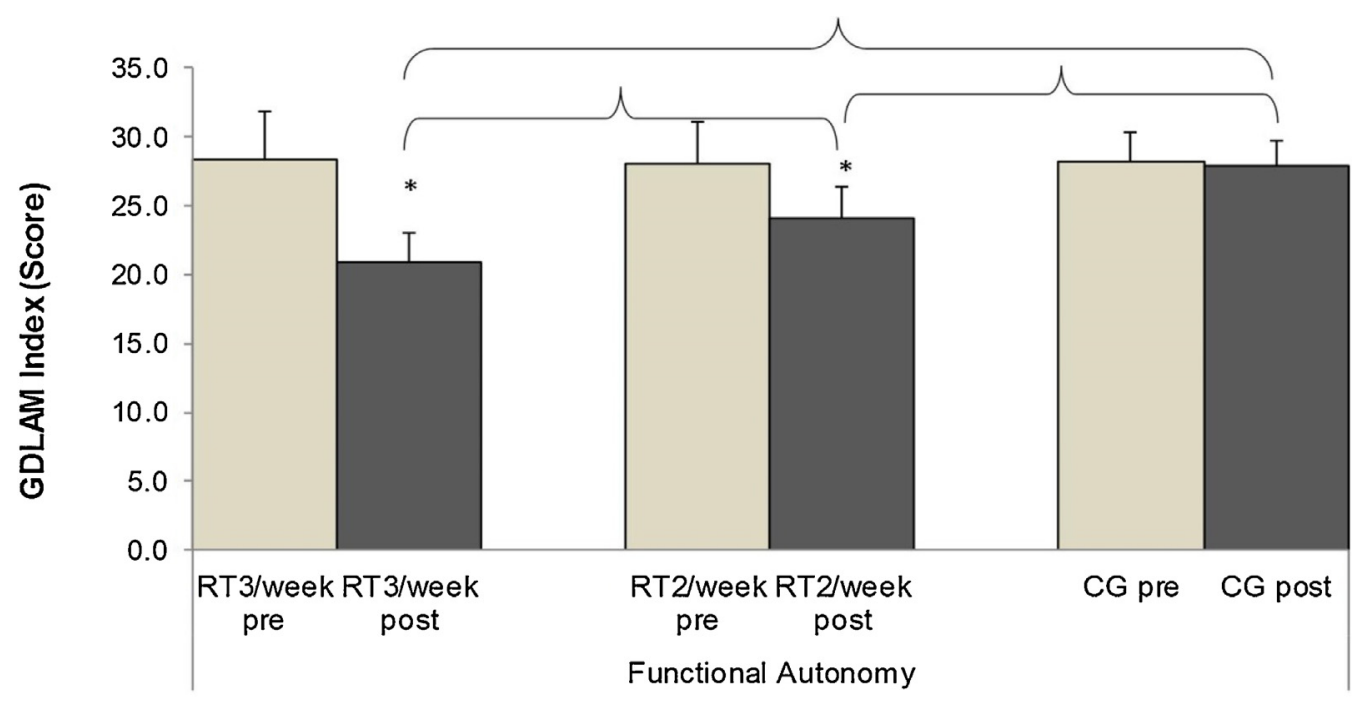

\section{Groups /Tests}

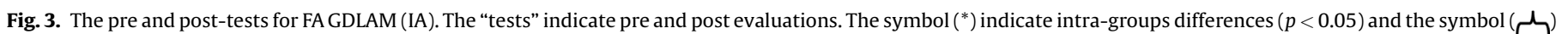
indicate inter-groups differences $(p<0.05)$. 


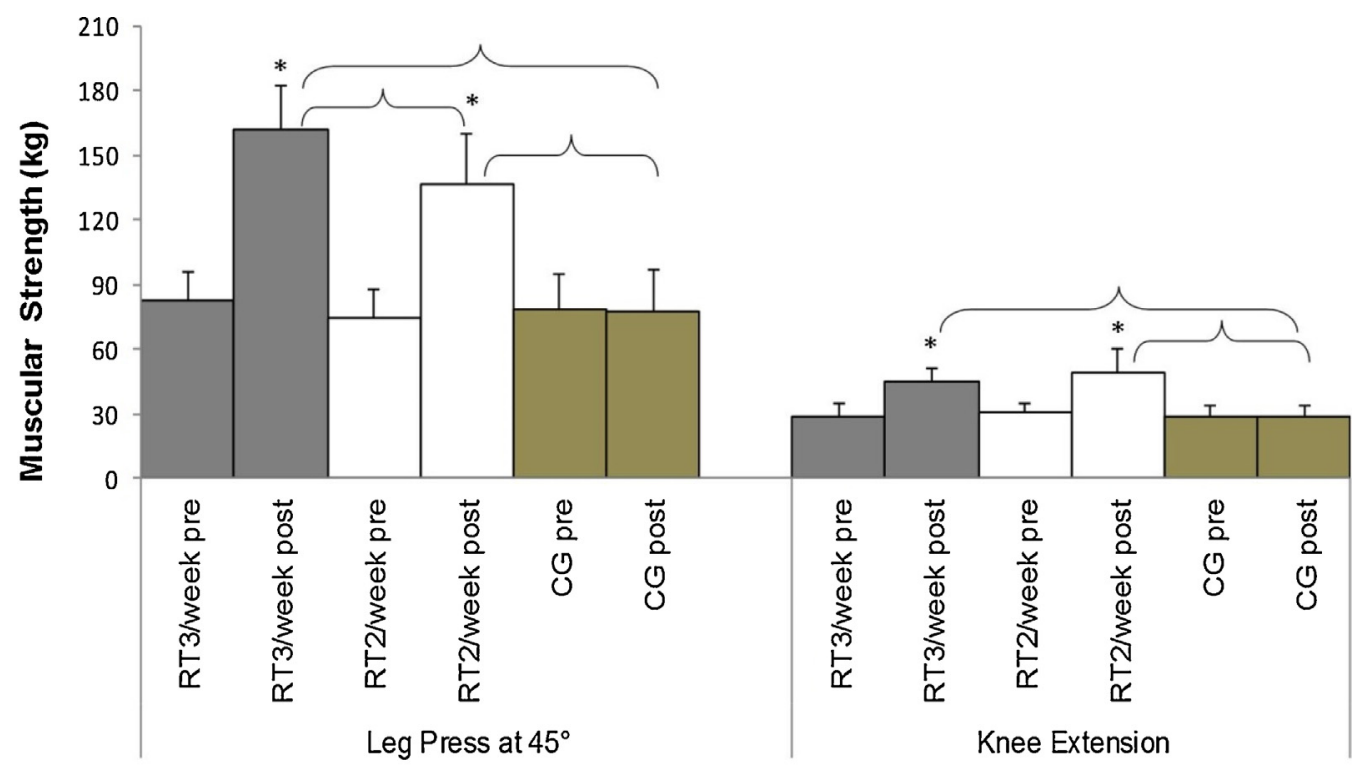

Groups / Exercises and Tests

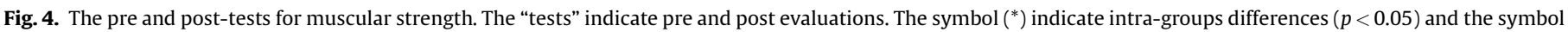
( indicate inter-groups differences $(p<0.05)$.

RT3 also showed better results $(\Delta \%=24.97 \%)$ compared with RT2. Also, both experimental groups $(p<0.05)$ : RT3 $(\Delta \%=84.1 \%)$ and RT2 $(\Delta \%=59.1 \%)$ showed better results compared with CG (Fig. 4$)$.

Fig. 4 also shows the results for knee extension and again both experimental groups were effective $(p<0.01$, intra-groups): RT3 $(\Delta \%=15.5 \%)$ and RT2 $(\Delta \%=18.8 \%)$. Also, the RT3 $(\Delta \%=15.28 \%)$ and RT2 $(\Delta \%=20.37 \%)$ were also better compared to the CG.

The results listed below are of OPAQ protocol showed statistical differences $(\mathrm{H}=461.07, p<0.0001)$ presented in Table 2 .

For total score OPAQ experimental groups showed improvement $(p<0.0001)$ intra-groups: RT3 $(\Delta \%=38.45 \%)$ and RT2 ( $\Delta \%=35.5 \%)$. The RT3 group also showed better results ( $\Delta \%=20.23 \%, \mathrm{p}=0.026$ ) compared to RT2. Moreover, both experimental groups: RT3 $(\Delta \%=57.61 \%)$ and RT2 $(\Delta \%=37.37 \%)$ presented better results $(p<0.0001)$ compared with CG. In the overall health, the intra-groups results were $\operatorname{similar}(p<0.0001)$ to the experimental groups: RT3 $(\Delta \%=5.55 \%)$ and RT2 $(\Delta \%=3.62 \%)$; also showed that RT3 $(\Delta \%=2.6 \%)$ and RT2 $(\Delta \%=3.25 \%)$ were showed compared with CG.

Both groups RT3 $(\Delta \%=2.55 \%)$ and RT2 $(\Delta \%=2.37 \%)$ showed intra-groups improvements $(p<0.05)$ for the mobility. Furthermore, the RT3 presented better result $(\Delta \%=3.46 \%, \mathrm{p}=0.001)$ compared with CG. The experimental groups: RT3 $(\Delta \%=16.5 \%)$ and RT2 $(\Delta \%=2.75 \%)$ also presented improvement $(p<0.05)$ for walk and lean compared to CG. In back pain, the experimental groups showed intra-groups improvements $(p<0.05)$ : RT3 $(\Delta \%=2.7 \%)$

Table 2

Present results for QoL variables of the studied groups.

\begin{tabular}{|c|c|c|c|c|c|c|c|}
\hline \multirow[t]{2}{*}{ Variables } & \multicolumn{2}{|l|}{ RT3, $n=20$} & \multicolumn{2}{|l|}{$\mathrm{RT} 2, n=16$} & \multicolumn{2}{|l|}{$\mathrm{CG}, n=16$} & \multirow[t]{2}{*}{ ES } \\
\hline & $\begin{array}{l}\text { Pre } \\
\text { Mean } \pm \text { SD }\end{array}$ & $\begin{array}{l}\text { Post } \\
\text { Mean } \pm \text { SD }\end{array}$ & $\begin{array}{l}\text { Pre } \\
\text { Mean } \pm \text { SD }\end{array}$ & $\begin{array}{l}\text { Post } \\
\text { Mean } \pm \text { SD }\end{array}$ & $\begin{array}{l}\text { Pre } \\
\text { Mean } \pm \text { SD }\end{array}$ & $\begin{array}{l}\text { Post } \\
\text { Mean } \pm \text { SD }\end{array}$ & \\
\hline Overall Health (points) & $20.3 \pm 2.5$ & $25.9 \pm 2.8^{*} \ddagger$ & $19.5 \pm 4.05$ & $23.1 \pm 2.1^{*} \dagger \dagger$ & $19.7 \pm 4.05$ & $19.8 \pm 4.1 \ddagger \dagger$ & 1.00 \\
\hline Mobility (points) & $21.3 \pm 2.4$ & $23.9 \pm 1.5^{*} \ddagger$ & $20 \pm 2.4$ & $22.4 \pm 2.1^{*}$ & $19.06 \pm 3.5$ & $20.4 \pm 4.8 \ddagger$ & 0.99 \\
\hline Walk and Lean (points) & $19.8 \pm 2.4$ & $21.6 \pm 2.2 \ddagger$ & $18.8 \pm 2.6$ & $20.4 \pm 2.1 \dagger$ & $16.1 \pm 3.6$ & $16.4 \pm 4.1 \ddagger \dagger$ & 1.00 \\
\hline Back Pain (points) & $17.9 \pm 2.7$ & $20.6 \pm 2.4^{*} \ddagger$ & $15.7 \pm 3.5$ & $19.7 \pm 2.3 * \dagger$ & $15.7 \pm 2.4$ & $15.5 \pm 4.1 \ddagger \dagger$ & 1.00 \\
\hline Flexibility (points) & $17.9 \pm 1.6$ & $19.1 \pm 1.1_{\ddagger}^{*}$ & $17 \pm 1.8$ & $18.8 \pm 1.8^{*} \dagger$ & $17.7 \pm 2.4$ & $17.2 \pm 2.4 \div \dagger$ & 0.90 \\
\hline Personal Health Care (points) & $19.4 \pm 0.8$ & $19.8 \pm 0.7$ & $19.1 \pm 0.9$ & $19.3 \pm 0.9$ & $19.4 \pm 0.9$ & $19.6 \pm 0.6$ & 0.51 \\
\hline House Chores (points) & $19.3 \pm 0.8$ & $19.5 \pm 0.6 \ddagger$ & $18.4 \pm 1.6$ & $19.3 \pm 0.6 \dagger$ & $18.5 \pm 1.8$ & $18.3 \pm 2.06 \ddagger \dagger$ & 0.84 \\
\hline Locomotion (points) & $19.2 \pm 1.06$ & $19.7 \pm 0.8 \ddagger$ & $18.6 \pm 1.1$ & $18.8 \pm 1.1 \ddagger$ & $19.4 \pm 1.09$ & $19 \pm 1.2 \ddagger$ & 0.78 \\
\hline Fear of Falls (points) & $17.8 \pm 2.9$ & $20.2 \pm 1.9 \ddagger$ & $16 \pm 4.6$ & $18.5 \pm 2.7 \dagger$ & $12.7 \pm 5.5$ & $11.8 \pm 5.2 \ddagger \dagger$ & 1.00 \\
\hline Social Activity (points) & $17.6 \pm 2.7$ & $19.1 \pm 2 \ddagger$ & $17.8 \pm 2.5$ & $18.7 \pm 2.3 \dagger$ & $16.9 \pm 2.8$ & $16.3 \pm 2.4 \neq \dagger$ & 0.86 \\
\hline Family Support (points) & $14.7 \pm 3.5$ & $16.4 \pm 3.4$ & $14.2 \pm 3.5$ & $15.8 \pm 3.5$ & $16.6 \pm 2.8$ & $16.2 \pm 2.9$ & 0.51 \\
\hline Osteoporosis Pain (points) & $17.6 \pm 3.8$ & $21.2 \pm 3.3^{*} \ddagger$ & $16.2 \pm 3.9$ & $18.3 \pm 3.3 \ddagger$ & $17.06 \pm 5$ & $17.1 \pm 5.2 \ddagger$ & 0.89 \\
\hline Sleep (points) & $12.8 \pm 3.8$ & $16.3 \pm 2.7^{*} \ddagger$ & $11.8 \pm 3.7$ & $15.5 \pm 2.7^{*} \dagger$ & $12.7 \pm 4.4$ & $12.8 \pm 4.3 \ddagger \dagger$ & 0.96 \\
\hline Fatigue (points) & $13 \pm 2.1$ & $16.2 \pm 2.1^{*} \ddagger$ & $12.7 \pm 2.6$ & $16 \pm 2.1^{*} \dagger$ & $12.9 \pm 2.9$ & $13.4 \pm 3.1 \ddagger \dagger$ & 0.99 \\
\hline Work (points) & $17.3 \pm 2.4$ & $18.9 \pm 1.6^{*}$ & $16.5 \pm 2.03$ & $17.7 \pm 1.8$ & $17.5 \pm 2.8$ & $17.8 \pm 2.5$ & 0.71 \\
\hline Tension Level (points) & $15.3 \pm 2.2$ & $17.6 \pm 2.3^{*}$ & $14.9 \pm 1.9$ & $17 \pm 2.5$ & $15.8 \pm 4.8$ & $15.5 \pm 4.4$ & 0.63 \\
\hline Humor (points) & $20.2 \pm 3.2$ & $21.4 \pm 2.4 \ddagger$ & $18.3 \pm 2.5$ & $19.2 \pm 2$ & $18.9 \pm 4.7$ & $18.8 \pm 4.7 \ddagger$ & 0.66 \\
\hline Body Image (points) & $13.4 \pm 4.5$ & $16.3 \pm 3.8^{*} \ddagger$ & $11.7 \pm 4.6$ & $15.1 \pm 3.7^{*}$ & $12.1 \pm 3.8$ & $12.4 \pm 4.1 \ddagger$ & 0.91 \\
\hline Independence (points) & $14.8 \pm 1.3$ & $15.1 \pm 0.3 \ddagger$ & $13.8 \pm 1.7$ & $14.7 \pm 0.8 \dagger$ & $13.2 \pm 1.8$ & $12.5 \pm 2.06 \ddagger \dagger$ & 1.00 \\
\hline Total OPAQ (score) & $330.2 \pm 22.02$ & $369.05 \pm 19.9 * \frac{9}{\ddagger}$ & $313.3 \pm 22.01$ & $348.8 \pm 22.6^{*} \dagger \ddagger$ & $312.3 \pm 35.9$ & $311.4 \pm 35.7 \ddagger \dagger$ & 1.00 \\
\hline
\end{tabular}

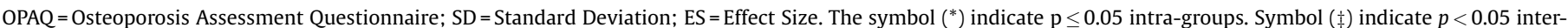
groups favorable RT3. And, symbol $(\dagger)$ indicate $p<0.05$ inter-groups favorable RT2. 
and RT2 $(\Delta \%=2.31 \%)$. The RT3 $(\Delta \%=5.03 \%)$ and RT2 $(\Delta \%=18.4 \%)$ were also better than the CG. A similar result $(p<0.05)$ occurred with the flexibility, because RT3 $(\Delta \%=1.2 \%)$ and RT2 $(\Delta \%=1.8 \%)$ showed improvements $(p<0.05)$ intra-groups and were also better RT3 $(\Delta \%=1.9 \%)$ and RT2 $(\Delta \%=1.62 \%)$ compared with CG.

The house chores, fear of falls and independence variables presented only inter-groups results. In these cases, RT2 and RT3 groups were better $(p<0.05)$ than CG: house chores (RT3 $\Delta \%=23.1 \%$; RT2 $\Delta \%=1 \%$ ); fear of falls (RT3 $\Delta \%=8.38 \%$; RT2 $\Delta \%=6.68 \%$ ) and Independence (RT3 $\Delta \%=2.53 \%$; RT2 $\Delta \%=2.18 \%$ ) compared with the CG. However, locomotion showed improvements $(p<0.05)$ only for RT3 compared with RT2 $(\Delta \%=0.88 \%)$ and CG $(\Delta \%=0.7 \%)$. For social activities both groups RT3 $(\Delta \%=2.78 \%)$ and RT2 $(\Delta \%=2.43 \%)$ were better $(p<0.05)$ than the CG. The osteoporosis pain showed improvement $(p<0.05)$ only for RT3 intra-group $(\Delta \%=3.6 \%)$ but also presented results intergroups $(p<0.05)$ compared to RT2 $(\Delta \%=2.93 \%)$ and with CG $(\Delta \%=18.4 \%)$.

Both groups RT3 $(\Delta \%=3.55 \%)$ and RT2 $(\Delta \%=3.62 \%)$ showed improvements $(p<0.05)$ intra-groups for sleep. Moreover, the RT3 $(\Delta \%=3.47 \%)$ and RT2 $(\Delta \%=2.62 \%)$ showed better results $(p<0.05)$ compared with CG. In the fatigue, the RT3 $(\Delta \%=3.15 \%)$ and RT2 $(\Delta \%=3.25 \%)$ showed intra-groups improvements $(p<0.05)$. And, TR3 $(\Delta \%=2.76 \%)$ and TR2 $(\Delta \%=2.56 \%)$ were also better $(p<0.05)$ than the CG. The work $(\Delta \%=1.6 \%)$ and tension level $(\Delta \%=2.27 \%)$ showed only improvements $(p<0.05)$ to RT3 intra-group. For humor, improvements occurred only in RT3 $(\Delta \%=2.58 \%, \mathrm{p}=0.025)$ compared with CG.

Finally, for the body image, the RT3 $(\Delta \%=2.95 \%)$ and RT2 $(\Delta \%=3.77 \%)$ showed improvements $(p<0.05)$ intra-groups. However, only RT3 showed better results $(\Delta \%=3.91 \%, \mathrm{p}=0.005)$ compared with CG.

\section{Discussion}

The literature has shown that PA can have a potential role in aiding or modifying the effects of osteoporosis (American College Sports Medicine (ACSM), 2014; Borba-Pinheiro et al., 2010; Lang et al., 2010). RT is the activity that generally shows the best results in the stimulation of osteogenesis and low BMD related-variables such as: FA, strength and QoL (Borba-Pinheiro et al., 2010; Cabral et al., 2014; Lang et al., 2010).

The BMD of all bone variables analyzed in this study were significantly better $(p<0.05)$ only for RT3 and also showed intergroup improvement $(p<0.05)$ compared with CG. Additionally, the RT2 showed improvement $(p<0.05)$ to the total BMD compared with GC. It is noteworthy that all groups had medical recommendation to consume specific medicine to control low BMD.

Borba-Pinheiro et al. (2010) are consistent with of results of the present study; significant differences $(p<0.05)$ were found for BMD in the lumbar spine in favor of the RT group, that also was realized three times week, with greater percentage gains in relation to the water exercise and CG. For the femoral neck site, the RT group was statistically better than water exercise and CG. In relation to the trochanteric site, the RT group was only better than the CG and did not differ from the other groups.

The muscle strength evaluated by leg press $45^{\circ}$ and knee extension in this study showed significant increases $(p<0.05)$ in the analyzed exercises for experimental intra-groups. The exercises leg press at $45^{\circ}$, knee extension, squats, hip adduction and machine for gluts no have direct action on the lumbar muscles. Though, can have effects piezoelectric effect (Boskey \& Coleman, 2010) for load pushed by the lower limbs that pressed on the lower back, causing isometric strength of the lumbar and abdominal muscles. Perhaps this can contribute to improvement in lumbar spine BMD.
The scientific literature (Filippin, Teixeira, Silva, Miraglia, \& Silva, 2014; Lang et al., 2010) reinforces the need to develop strength in the elderly because the muscle hypertrophy coincide with significant elevations of attenuated muscle IGF levels, revealing a possible contributory mechanism for sarcopenia control. There is a positive relationship between the increase in muscular strength and BMD (Borba-Pinheiro et al., 2010; Rubin et al., 2006). Studies have suggested that periodized RT programs have a greater possibility for effective strength gains (American College Sports Medicine (ACSM), 2014), a hypothesis can be formulated that this type of training would also be the most suitable to increase BMD (Lang et al., 2010).

However, body composition variables were entered as the most significant contributors for BMD and strength. Although, functional fitness parameters are typically considered in clinical assessments of bone health/strength in older people; although, body composition appears to have a higher relevance in the explanation of BMD and strength (Gouveia et al., 2012). In present study, the baseline BMI and body mass showed statistics differences intergroups that can evidence an increase for care with falls and greater need of intervention by exercise training.

Park et al. (2013) evaluated changes in muscle mass and strength according to changes in BMD after alendronate-calcitriol therapy and also to assessed subsequent changes in common biomarkers for osteoporosis and sarcopenia, showed improvement in lumbar BMD and in handgrip strength observed after 6 months of alendronate-calcitriol combination therapy. Initial serum interleukin-6 levels (IL-6) presented an inverse relationship with pretreatment lumbar BMD and handgrip strength. The degree of change in IL-6 levels induced by the combination therapy was correlated with the initial degree of the catabolic status of the bone markers, such as parathyroid hormone and the severity of lumbar BMD derangements that are considered a representative parameter for sarcopenia.

Another study with 164 menopausal women compared four groups: Group 1 (G1) subjects treated with $5 \mathrm{mg}$ alendronate/day plus jumping exercises with progressive effort; Group 2 (G2) subjects treated only with $5 \mathrm{mg}$ alendronate/day; Group 3 (G3) subjects treated with placebo plus progressive jumping exercise; and Group 4 (G4) subjects treated only with placebo. It was observed that G1 had a significant improvement in the BMD of the femur and lumbar spine compared with G3. However, the latter had a significant improvement (3.6\%) in the distal tibia when compared with those that did not practice PA (G2 and G4) (UusiRasi et al., 2003).

The drug therapy with bisphosphonate is recommended and safe for beneficial effects on bone health in postmenopausal women, especially, when there is an increased risk of fracture in hip and lumbar spine (Diab \& Watts, 2013; Reginster, 2011). According to a study of Mukaiyama et al. (2015) the combined effect of alendronate with alfacalcidol and eldecalcitol (analogous to vitamin D), showed significant improvement in BMD of the lumbar spine, maintenance the femur neck and showed no improvement in hip BMD after one year of treatment.

The present study utilized $70 \mathrm{mg}$ sodium alendronate and vitamin D3 5600 IU concomitant in 20 osteoporotic postmenopausal women; and only vitamin D3 in 32 osteopenic postmenopausal women distributed in the groups: RT3, RT2 and CG. In the GC not was observed increase BMD $(p<0.05)$ in the variables, characterized as a BMD maintenance. Although the drug was not analyzed as an independent variable, it was likely that the drug can have contributed to the increase or maintenance of the BMD, because, there is evidence that a combination of PA with alendronate shows positive effects on BMD (Mukaiyama et al., 2015; Uusi-Rasi et al., 2003). Therefore, together, alendronate and 
PA can be more efficient in reducing the risk of fracture (BorbaPinheiro et al., 2010; Uusi-Rasi et al., 2003).

The results of QoL for this study showed that experimental groups were effective for most variables of the OPAQ protocol. In terms of effects on QoL, (2010) showed that QoL of RT group presented significant improvement $(p<0.05)$ in comparison to the CG, but it did not show improvements in relation to the others experimental groups of postmenopausal women: water exercise and adapted judo training.

QoL is impaired in women with low BMD, as shown in the study by Romagnoli et al. (2004) that compared the QoL of 361 women, either normal or with osteopenia or osteoporosis. In this study, the women with osteoporosis, with or without fractures, showed a significant reduction $(p<0.05)$ in the perception of general health, physical and social function and total count. Subjects with spinal fractures and low femoral BMD had impaired perception of QoL. These results were confirmed by Adachi et al. (2002), who evaluated the QoL of women over 50 years with low BMD that suffered from spinal or no spinal fractures or no fractures and found a worsening of QoL measures in the groups with spinal and no spinal fractures.

Another variable assessed in this study was FA. The functional capacity of physically active older adult women is considered statistically better - regarding autonomy tests and the GDLAM index - when compared to sedentary older adult women's functional capacity (Guido et al., 2010; Santos \& Griep, 2013). Studies show that PA can increase and maintain functional independence levels in physically active older adults, especially for the performance of activities of daily living (Daniel, Vale, Giani, Bacelar, \& Danats, 2012; Pernambuco et al., 2013).

The results of this study for FA in the experimental groups showed improvement $(p<0.05)$. The results reinforce the studies cited above, showing that the planned exercise has the potential to improve FA of older women. The studies also show the need to maintain FA, because the loss of this capacity compromises basic personal care activities, such as brushing teeth, showering, putting on shoes, and dressing up, among others, causing greater dependency to older adults, besides needing care provided by third parties (Guido et al., 2010; Cabral et al., 2014). These studies show to the Brazilian public basic healthcare that PA of RT can be part of care programs directed to the older adult population in the public health network.

The results of the present study corroborate those reported in the literature for periodized RT programs, because they can be effective in the BMD, strength, FA and QoL (American College Sports Medicine (ACSM), 2014; Borba-Pinheiro et al., 2010). In addition, these results are in agreement with the recommendations of Lang et al. (2010) because they claim that RT programs show significant increases in muscle strength of the lower limbs, contributing to sarcopenia and BMD controls which helps to maintain mobility, decreasing falls and consequently fractures risk in the elderly. It is therefore, suggested that PAs programs for osteoporotic patients follow ACSM guidelines promoting strength development. Nevertheless, they must also meet the needs of flexibility, balance, strength and FA, since these variables contribute to reducing risk factors for falls, enabling the elderly to enjoy an active lifestyle (American College Sports Medicine (ACSM), 2014; Gouveia et al., 2012).

Although, studies show the benefits of exercise for BMD and health-variables of postmenopausal women (Borba-Pinheiro et al., 2010; Gouveia et al., 2013). The sample size has been a problem reported in the literature for studies involving exercise and skeletal structure, because the potentially beneficial effects for BMD, geometry, bone strength and fractures in human weakened by a small sample size (Kemmler, Häberle, \& Von Stengel, 2013; Polidoulis, Beyene, \& Cheung, 2012). The difficulty of researchers to maintain a large number of elderly volunteers for a long period can be one of these difficulties. This was observed in the present study.

The limitations of this study include the non-use of alendronate, vitamin $\mathrm{D}^{+}$and nutritional dietary as independent variables, besides, the small sample size. Therefore, additional studies are recommended.

\section{Conclusion}

In conclusion, the study showed that both experimental groups presented favorable results for BMD, strength, FA and QoL. However, the RT3 showed the best results compared to other groups after 13 months of intervention.

\section{Conflict of interest}

All authors declared did not have financial support of any institution, so there are no potential conflicts of interest for this study.

\section{Acknowledgements}

To National Council for Scientific and Technological Development of Brazil (CNPq/Brasil).

\section{References}

Adachi, J. D., Ioannidis, G., Olszinski, W. P., Brown, J. P., Hanley, D. A., Sebaldt, R. J., et al. (2002). The impact of incident vertebral, non-vertebral fractures on health related quality of life in postmenopausal women. BMC Musculoskeletal Disorders, $3,11$.

Albright, F., Burnett, C. H., Cope, O., \& Parson, W. (1941). Acute atrophy of bone (osteoporosis) simulating hyperparathyroidism. Journal of Clinical Endocrinology and Metabolism, 1, 711-716.

American College Sports Medicine (ACSM) (2014). Guidelines for exercise testing and prescription, 9 ed. Rio de Janeiro: Guanabara (in Portuguese).

Barreto, A. C. L. Y. G., \& Ribeiro, L. G. (2004). Determining the sample size. Fitness $\mathcal{E}$ Performance Journal, 3(3), 124 (in Portuguese).

Borba-Pinheiro, C. J., Carvalho, M. C. G. A., Silva, N. S. L., Drigo, A. J., Bezerra, J. C., \& Dantas, E. H. M. (2010). Bone density, balance and quality of life of postmenopausal women taking alendronate, participating in different physical activity programs. Therapeutic Advances in Musculoskeletal Disease, 2, 175-185.

Boskey, A. L., \& Coleman, R. (2010). Aging and Bone. Journal of Dental Research, 89 (12), 1333-1348

Cabral, A. C. A., Magalhães, I. K. M., Borba-Pinheiro, C. J., Rocha-Júnior, O. B. M. Figueire, N. M. A., \& Dantas, E. H. M. (2014). Body composition and functional autonomy of older adult women after a resistance training program. Journal of Research: Fundamental Care Online, 6(1), 74-85. http://dx.doi.org/10.9789/21755361.2014v6n1p74.

Cantarelli, F. B., Szejnfeld, V. L., Oliveira, L. M., Ciconelli, R. M., \& Ferraz, M. B. (1999). Quality of life in patients with osteoporosis fractures: cultural adaptation, reliability and validity of the Osteoporosis Assessment Questionnaire. Clinical and Experimental Rheumatology, 17, 547-551.

Cusller, E. C., Going, S. B., Houtkooper, L. B., Stanford, V. A., Blew, R. M., Flint-Wagner, H. G., et al. (2005). Exercise frequency and calcium intake predict-4year bone changes in postmenopausal women. Osteoporosis International, 16, 2129-2141.

Dantas, E. H. M., Figueira, A. H., Emygdio, R., \& Vale, R. S. (2014). Functional autonomy gdlam protocol classification pattern in elderly women. Indian Journal of Applied Research, 4(7), 262-266.

Daniel, F. N. R., Vale, R. G. S., Giani, T. S., Bacelar, S., \& Danats, E. H. M. (2012) Functional autonomy of older women participate of a physical activities program. Acta Scientiarum Health Science, 34, 151-156 (in Portuguese).

Diab, D. L., \& Watts, N. B. (2013). Bisphosphonate drug holiday: who, when and how long. Therapeutic Advances in Musculoskeletal Disease, 5(3), 107-111. http://dx. doi.org/10.1177/ 1759720X13477714

Filippin, L. I., Teixeira, V. N. O., Silva, M. P. M., Miraglia, F., \& Silva, F. S. (2014). Sarcopenia: a predictor of mortality and the need for early diagnosis and intervention. Aging Clinical and Experimental Research. http://dx.doi.org/ 10.1007/s40520-014-0281-4.

Gouveia, E. R., Maia, J.Á., Beunen, G. P., Blimkie, C. J., Rodrigues, A. L., \& Freitas, D. L. (2012). Functional fitness and bone mineral density in the elderly. Archives of Osteoporosis, 7(1), 75-85. http://dx.doi.org/10.1007/s11657-012-0083-2.

Guido, M., Lima, R. M., Benford, R., Leite, T. K. M., Pereira, R. W., \& Oliveira, R. C. (2010). Effects of 24 weeks of resistance training on aerobic fitness indexes of older women. The Revista Brasileira de Medicina do Esporte, 16(4), 259-263 (in Portuguese). 
Gouveia, E. R., Maia, J.Á., Beunen, G. P., Blimkie, C. J., Fena, E. M., \& Freitas, D. L. (2013) Functional fitness and physical activity of Portuguese community-residing older adults. Journal of Aging and Physical Activity, 21(1).

Kanis, J. A., McCloskey, E. V., Johansson, H., Cooper, C., Rizzoli, R., \& Reginster, J.-Y. (2013). European guidance for the diagnosis and management of osteoporosis in postmenopausal women: Position paper. Osteoporosis International, 24, $23-$ 57. http://dx.doi.org/10.1007/s00198-012-2074-y.

Kemmler, W., Häberle, L., \& Von Stengel, S. (2013). Effects of exercise on fracture reduction in older adults: a systematic review and meta-analysis. Osteoporosis International, 24(7), 1937-1950. http://dx.doi.org/10.1007/s00198-012-2248-7.

Kraemer, W., \& Fleck, S. J. (2009). Optimizing strength training: designing nonlinear periodization workouts. Barueri, SP: Manole (in Portuguese).

Lang, T., Streeper, T., Cawthon, P., Baldwin, K., Taaffe, D. R., \& Harris, T. B. (2010). Sarcopenia: etiology, clinical consequences, intervention, and assessment. Osteoporosis International, 21, 543-559. http://dx.doi.org/10.1007/s00198-0091059-y.

Langdahl, B. L. H. T. (2011). Medical treatment of osteoporotic vertebral fractures. Therapeutic Advances in Musculoskeletal Disease, 3(1), 17-29.

Mukaiyama, K., Uchiyama, S., Nakamura, Y., Ikegami, S., Taguchi, A., Kamimura, M., et al. (2015). Eldecalcitol, in combination with bisphosphonate, is effective for treatment of Japanese osteoporotic patients. The Tohoku Journal of Experimental Medicine, 237(4), 339-343. http://dx.doi.org/10.1620/tjem.237.339.

Park, J. H., Park, K. H., Cho, S., Choi, Y. S., Seo, S. K., Lee, B. S., et al. (2013). Concomitant increase in muscle strength and bone mineral density with decreasing IL6 levels after combination therapy with alendronate and calcitriol in postmenopausal women. Menopause, 20(7), 747-753. http://dx.doi.org/ 10.1097/GME.0b013e31827cabca.

Patel, S., Tweed, K., \& Chinappen, U. (2005). Fall-related risk factors and osteoporosis in older women referred to an open access bone densitometry service. Age and Ageing, 34, 67 10.1093/ageing/afh238.

Pernambuco, C. S., Borba-Pinheiro, C. J., Vale, R. G. S., Di Masi, F., Monteiro, P. K. P., \& Dantas, E. H. M. (2013). Functional autonomy, bone mineral density (BMD) and serum osteocalcin levels in older female participants of an aquatic exercise program (AAG). Archives of Gerontology and Geriatrics, 56, 466-471.

Pinheiro, M. M., Ciconelli, R. M., Jacques, N. O., Genaro, P. S., Martini, L. A., \& Ferraz, M. B. (2010). he impact of osteoporosis in Brazil: regional data from fractures in adult men and women-The Brazilian Osteoporosis Study (BRAZOS). Revista Brasileira de Reumatologia, 50(2), 113-127.

Pinheiro, M. M., Ciconelli, R. M., Martini, L. A., \& Ferraz, M. B. (2010). Risk factors for recurrent falls among Brazilian women and men: the Brazilian Osteoporosis Study (BRAZOS). Cadernos de Saúde Pública, 26(1), 89-96.

Polidoulis, I., Beyene, J., \& Cheung, A. M. (2012). The effect of exercise on pQCT parameters of bone structure and strength in postmenopausal women-a systematic review and meta-analysis of randomized controlled trials. Osteoporosis International, 23(1), 39-51. http://dx.doi.org/10.1007/s00198-0111734-7.

Reginster, J. Y. (2011). Antifracture efficacy of currently available therapies for postmenopausal osteoporosis. Drugs, 71(1), 65-78. http://dx.doi.org/10.2165/ 11587570-000000000-00000.

Romagnoli, E., Carnevale, V., Nofroni, I., D’erasmo, E., Paglia, F., De Geronimo, S., et al (2004). Quality of life in ambulatory postmenopausal women: the impact of reduced bone mineral density hand subclinical vertebral fractures. Osteoporosis International, 15, 975-980.

Rubin, C., Judex, S., \& Qin, Y.(2006). Low-level mechanical signals and their potential as a non-pharmacological intervention for osteoporosis. Age and Ageing, 35 (Suppl. 2), ii32-ii36. http://dx.doi.org/10.1093/ageing/afl082.

Santos, M. I. P. O., \& Griep, R. H. (2013). Functional capacity of the elderly attended in a unified health system (SUS) program in Belém in the State of Pará. Ciência E Saúde Coletiva, 18(3), 753-761 (in Portuguese).

Uusi-Rasi, K., Kannus, P., Cheng, S., Sievanen, H., Pasanen, M., Heinonen, A., et al. (2003). Effect of alendronate and exercise on bone and physical performance of postmenopausal women: a randomized controlled trial. Bone, 33, 132-143.

World Medical Association (2008). Declaration of helsinki. Ethical principles for medical research involving human subjects. 59th WMA general assembly. 